\title{
High FNDC1 expression correlates with poor prognosis in gastric cancer
}

\author{
MUXIAO ZHONG ${ }^{1 *}$, YIJIE ZHANG ${ }^{1 *}$, FANGFANG YUAN $^{2}$, YAO PENG $^{3}$, \\ JINGJING WU ${ }^{1}$, JIAWEI YUAN ${ }^{1}$, WEI ZHU ${ }^{1}$ and YALI ZHANG ${ }^{1}$
}

\begin{abstract}
${ }^{1}$ Guangdong Provincial Key Laboratory of Gastroenterology, Department of Gastroenterology, Nanfang Hospital, Southern Medical University, Guangzhou, Guangdong 510515; ${ }^{2}$ Department of Intensive Care Unit, General Hospital of Guangzhou Military Command, Guangzhou, Guangdong 510010; ${ }^{3}$ Department of Gastroenterology, Guangzhou Digestive Disease Center, Guangzhou First People's Hospital, Guangzhou Medical University, Guangzhou, Guangdong 510180, P.R. China
\end{abstract}

Received March 5, 2018; Accepted August 2, 2018

DOI: $10.3892 /$ etm.2018.6731

\begin{abstract}
Gastric cancer is a common human cancer worldwide. Fibronectin is an important extracellular matrix protein that has been implicated in many cancers and is known to be associated with proliferation and migration. Fibronectin type III domain containing 1 (FNDC1) contains a major component of the structural domain of fibronectin. The objectives of the present study were to measure FNDC1 expression in gastric cancer tissues and evaluate its value as a potential prognostic marker for gastric cancer. FNDC1 protein expression was analyzed by immunohistochemistry in 98 samples of gastric cancer tissue and 25 adjacent normal tissues. The associations between FNDC1 level and various clinicopathological characteristics were assessed, and the correlation between FNDC1 expression levels and prognosis of patients with gastric cancer was analyzed using a Kaplan-Meier analysis. It was demonstrated that FNDC1 expression in gastric cancer tissues and adjacent tissues was significantly different. FNDC1 expression levels were significantly higher in gastric cancer tissues compared with normal gastric tissues $(\mathrm{P}<0.001)$. Among the clinicopathological characteristics evaluated, clinical stage $(\mathrm{P}<0.001)$, $\mathrm{T}$ classification $(\mathrm{P}<0.001)$, $\mathrm{N}$ classification $(\mathrm{P}<0.001)$ and pathological differentiation $(\mathrm{P}=0.044)$ were significantly associated with high FNDC1 expression. Higher FNDC1 expression level was significantly
\end{abstract}

Correspondence to: Professor Yali Zhang, Guangdong Provincial Key Laboratory of Gastroenterology, Department of Gastroenterology, Nanfang Hospital, Southern Medical University, 1838 North of Guangzhou Avenue, Guangzhou, Guangdong 510515, P.R. China

E-mail: zyl41531@163.com

${ }^{*}$ Contributed equally

Key words: fibronectin type III domain containing 1, gastric cancer, prognosis, immunohistochemistry, protein expression correlated with poorer survival. The present findings suggest that FNDC1 expression levels may be a promising prognostic biomarker for gastric cancer.

\section{Introduction}

Despite the development of therapeutics in recent years, gastric cancer is one of the most recurrent malignant types of cancer and the second most common cause of cancer deaths worldwide (1). Of all reported cases, two-thirds of gastric cancer-related deaths have occurred in developing countries with high-risk areas, including China, Japan and Central and South America $(2,3)$. Several factors are known to serve important roles in promoting gastric cancer, including familial genetics and environmental factors, such as Helicobacter pylori infection, foods with high salt content and smoking (4-7). Irrespective of traditional treatments such as surgery, radiotherapy, and chemotherapy as well as novel cancer-targeting therapies, the overall 5-year survival rate in patients with gastric cancer is very low $(8,9)$. Thus, gastric cancer is a multi-factorial, complex disease, and the detailed mechanisms regulating its development and progression remain unclear. For these reasons, the identification of additional biomarkers of gastric cancer and therapeutic targets is imperative and necessary for improving the clinical outcome.

Tumor invasion and metastasis require proteolytic degradation of the basement membrane and the extracellular matrix (ECM). Fibronectin is an important ECM protein, which has been implicated in many cancers and is known to be associated with cancer proliferation and migration (10-13). Fibronectin type III domain containing 1 (FNDC1), also known as AGS8, contains a major component of the structural domain of fibronectin (13). Recent studies have demonstrated that FNDC1 is closely associated with the development of many diseases (14-21); however, the function of FNDC1 is still not clear. These studies demonstrated that FNDC1 expression increased with skin tumor progression and increasing tumor thickness (14). FNDC1 was demonstrated to be hypermethylated in adenoid cystic carcinoma (15). In vitro, knockdown of FNDC1 could suppress the cellular 
proliferation and migration of prostate cancer, while inducing apoptosis (16). van Ingen et al (17) performed a genome-wide association study and identified that FNDC1 serves an important role as a disease-contributing gene of acute otitis media in children. FNDC1 is also expressed in the kidney, may regulate $\mathrm{G}$ protein signaling and has been implicated in the hypoxia-induced apoptosis of cardiomyocytes (18-20). Recently, FNDC1 was reported to be associated with vascular endothelial growth factor (VEGF)-mediated cellular events, including tube formation, migration and proliferation (21). To date, however, the role of FNDC1 in the development and progression of gastric cancer has not been evaluated.

In the present study, the expression pattern of FNDC1 and correlations between its expression and the clinical characteristics of gastric cancer were investigated for the first time, to the best of our knowledge.

\section{Patients and methods}

Patients and tumor samples. Formalin-fixed tumor tissues from 98 patients ( 74 males, 24 females; age range, 25-83 years) including 25 paired adjacent normal tissues $(5 \mathrm{~cm}$ from tumor edges) were used for immunohistochemical analysis. All patients who underwent surgical resection for primary gastric cancer in the Department of General Surgery in Nanfang Hospital (Guangzhou, China) from March 2010 to December 2014 were enrolled in the present study. No patients had received radiotherapy or chemotherapy prior to surgical resection. Patients with any other types of cancer, or who missed follow-up appointments were excluded from the present study. Each tumor was assigned a histological type and a depth grading of infiltration according to the World Health Organization classification (22). The differentiation grade and Tumor, Node and Metastasis staging of gastric carcinoma were performed according to the AJCC Cancer Staging Manual (23). Diagnosis was established by two independent pathologists. The clinicopathological information of the 98 patients with gastric cancer is presented in Table I. All the tissue specimens for the present study were obtained from patients following provision of written informed consent. The tissue samples obtained from the tissue bank at Nanfang Hospital and this retrospective analysis were approved by the Ethics Committee of Nanfang Hospital.

Statistical analysis of FNDC1 expression in gastric cancer. To determine the expression pattern of FNDC1 in gastric cancer, datasets from the Oncomine database (https://www.oncomine.org) were used. FNDC1 was queried in the database, and the results were filtered by selecting gastric cancer and cancer vs. normal analysis. The Cho, Derrico, and Wang datasets obtained from the Oncomine database were embedded in the NCBI GEO database (https://www.ncbi.nlm. nih.gov/geo/) at accession numbers GSE13861, GSE13911 and GSE19826, respectively (24-26). The Cancer Genome Atlas (TCGA) gastric database was analyzed by GEPIA (http://gepia.cancer-pku.cn), a web-based tool to deliver fast and customizable functionalities based on TCGA and GTEx data (27). The following settings were used for the analysis: 'Expression on Box Plots'; 'Gene=FNDC1'; ' $\log 2 \mathrm{FCl}$ Cutoff=1'; 'P-value Cutoff=0.01'; 'Datasets=STAD'; 'Log Scale=Yes';
'Jitter Size=0.4'; and 'Match TCGA normal and GTEx data'. The prognostic value of the FNDC1 gene in gastric cancer was also analyzed using the Kaplan-Meier Plotter (http://kmplot. com/analysis/). The following settings were used for the analysis: 'Overall survival'; 'Post progression survival'; 'auto select best cutoff'; 'censore at threshold' (patients surviving over the selected threshold are censored instead of excluded); 'tumor stage all'; 'tumor stage $\mathrm{T}$ all'; 'tumor stage $\mathrm{N}$ all'; 'tumor stage M all'; 'Lauren classification all'; and 'differentiation all'. The FNDC1 gene probe set was 226930_at, and patients were split according to median expression or expression at best cutoff for the probe. The data were extracted from the Oncomine database, GEPIA website and Kaplan-Meier Plotter between December 2017 and March 2018.

Immunohistochemistry analysis. Immunohistochemistry analysis was performed as previously described. Formalin-fixed (fixed using 4\% formalin at room temperature for $24 \mathrm{~h}$ ) and paraffin-embedded tissues were cut into 4- $\mu \mathrm{m}$-thick sections, followed by incubation at $65^{\circ} \mathrm{C}$ for $2 \mathrm{~h}$. Tissues were deparaffinized in xylene and then rehydrated in graded alcohol and PBS. Following antigen retrieval by EDTA pre-incubated with $5 \%$ normal bovine serum (Wuhan Boster Biological Technology Ltd., Wuhan, China) at room temperature for $20 \mathrm{~min}$, deparaffinized sections were incubated overnight at $4^{\circ} \mathrm{C}$ with an optimal dilution (1:100) of a primary polyclonal rabbit antibody against human FNDC1 (abs127634a; Absin Bioscience, Inc., Shanghai, China). Following washing, the slides were incubated with horseradish peroxidase conjugated-anti-rabbit $\operatorname{IgG}$ secondary antibodies (1:200; cat. no. TA130023; OriGene Technologies, Inc., Beijing, China). Then, reaction products were treated with diaminobenzidine (DAB; Dako; Agilent Technologies, Inc., Santa Clara, CA, USA), counterstained with hematoxylin at room temperature for $5 \mathrm{~min}$, dehydrated, and mounted. For negative controls, the primary antibodies were omitted, but otherwise the methodology was the same. The Olympus BX51 microscope (Olympus Corporation, Tokyo, Japan) was used to capture images of the samples (magnification, x200 and x400). The sections were reviewed and scored independently by two observers, based on both the proportion of positively stained tumor cells and the intensity of staining. The proportion of positive tumor cells was scored as follows: 0 (no positive tumor cells), 1 ( $<10 \%$ positive tumor cells), 2 (10-50\% positive tumor cells), and 3 (>50\% positive tumor cells). The intensity of staining was graded according to the following criteria: 0 (no staining); 1 (weak staining=light yellow), 2 (moderate staining=yellow-brown), and 3 (strong staining=brown). The staining index was calculated as staining intensity score $x$ proportion of positive tumor cells. An optimal cut-off value was identified based on previous studies: A score of $\geq 4$ was defined as high FNDC1 expression and a score of $\leq 3$ was defined as low FNDC1 expression (28-31).

Statistical analysis. SPSS 13.0 software (SPSS, Inc., Chicago, IL, USA) was used for statistical analyses. Data of FNDC1 expression level obtained from the Oncomine database were analyzed using Student's t-test. The correlation between FNDC1 expression and clinicopathological parameters was measured by Pearson's $\chi^{2}$ test. Overall survival curves were estimated by the Kaplan-Meier method and log-rank test. Univariate and multivariate Cox regression survival analysis was performed 
Table I. Association between clinicopathological characteristics of gastric cancer and FNDC1 expression levels.

FNDC1 expression

\begin{tabular}{|c|c|c|c|c|c|}
\hline \multirow[b]{2}{*}{ Characteristic } & \multirow[b]{2}{*}{ Total } & & \multirow[b]{2}{*}{$\chi^{2}$} & \multirow[b]{2}{*}{ P-value } \\
\hline & & Low & High & & \\
\hline \multicolumn{6}{|l|}{ Age (years) } \\
\hline$<60$ & 53 & 13 & 40 & 0.528 & 0.467 \\
\hline$\geq 60$ & 45 & 14 & 31 & & \\
\hline \multicolumn{6}{|l|}{ Sex } \\
\hline Male & 74 & 23 & 51 & 1.886 & 0.170 \\
\hline Female & 24 & 4 & 20 & & \\
\hline \multicolumn{6}{|c|}{ Tumor size (diameter in $\mathrm{cm}$ ) } \\
\hline$<5$ & 51 & 15 & 36 & 0.184 & 0.668 \\
\hline$\geq 5$ & 47 & 12 & 35 & & \\
\hline \multicolumn{6}{|l|}{ Clinical stage } \\
\hline I & 7 & 7 & 0 & 26.48 & $<0.001$ \\
\hline II & 11 & 6 & 5 & & \\
\hline III & 62 & 11 & 51 & & \\
\hline IV & 18 & 3 & 15 & & \\
\hline \multicolumn{6}{|l|}{$\mathrm{T}$ classification } \\
\hline $\mathrm{T} 1+\mathrm{T} 2$ & 11 & 8 & 3 & 12.669 & $<0.001$ \\
\hline $\mathrm{T} 3+\mathrm{T} 4$ & 87 & 19 & 68 & & \\
\hline \multicolumn{6}{|l|}{$\mathrm{N}$ classification } \\
\hline N0 & 24 & 15 & 9 & 19.745 & $<0.001$ \\
\hline $\mathrm{N} 1$ & 16 & 2 & 14 & & \\
\hline $\mathrm{N} 2$ & 15 & 2 & 13 & & \\
\hline N3 & 43 & 8 & 35 & & \\
\hline \multicolumn{6}{|l|}{ Metastasis } \\
\hline No & 80 & 25 & 55 & 2.986 & 0.084 \\
\hline Yes & 18 & 2 & 16 & & \\
\hline \multicolumn{6}{|c|}{ Pathologic differentiation } \\
\hline Well & 5 & 1 & 4 & 6.247 & 0.044 \\
\hline Moderate & 32 & 14 & 18 & & \\
\hline Poor & 61 & 12 & 49 & & \\
\hline
\end{tabular}

Data are presented as number of patients (n). FNDC1, fibronectin type III domain containing 1.

to determine the independent prognostic markers. $\mathrm{P}<0.05$ was considered statistically significant.

\section{Results}

Analysis of FNDC1 gene expression. Data for FNDC1 gene expression were extracted from the Oncomine database and TCGA gastric database for gastric cancer, focusing on cancer vs. normal patient datasets. As presented in Fig. 1, FNDC1 mRNA expression in gastric cancer was demonstrated to be significantly upregulated in tumor tissues compared with normal tissues in the Cho, Derrico, and Wang datasets (Fig. 1A-C, respectively) as well as in the TCGA database analyzed by GEPIA (Fig. 1D).

High expression of FNDC1 in human gastric cancer tissues. The clinicopathological features of all 98 patients with gastric cancer are summarized in Table I. The expressed FNDC1 was detected in the nucleus and cytoplasm of the cancer cells, and FNDC1 was predominately localized in the nucleus (Fig. 2). FNDC1 was expressed in 12\% (3/25) of paired adjacent normal tissues. Compared with these normal tissues, a relatively high FNDC1 expression level was observed in $72.4 \%$ (71/98) of gastric cancer tissues and $27.6 \%$ (27/98) of cases exhibited relatively low FNDC1 expression (Table I).

Upregulation of FNDC1 is associated with advanced clinicopathological features of gastric cancer. To investigate the role of FNDC1 in gastric cancer, its expression was examined using immunohistochemistry in 98 paraffin-embedded archived human gastric cancer tissues, including 7 cases at clinical stage I, 11 cases at clinical stage II, 62 cases at clinical stage III, and 18 cases at clinical stage IV (Table I). As presented in Table I, significant associations were observed 


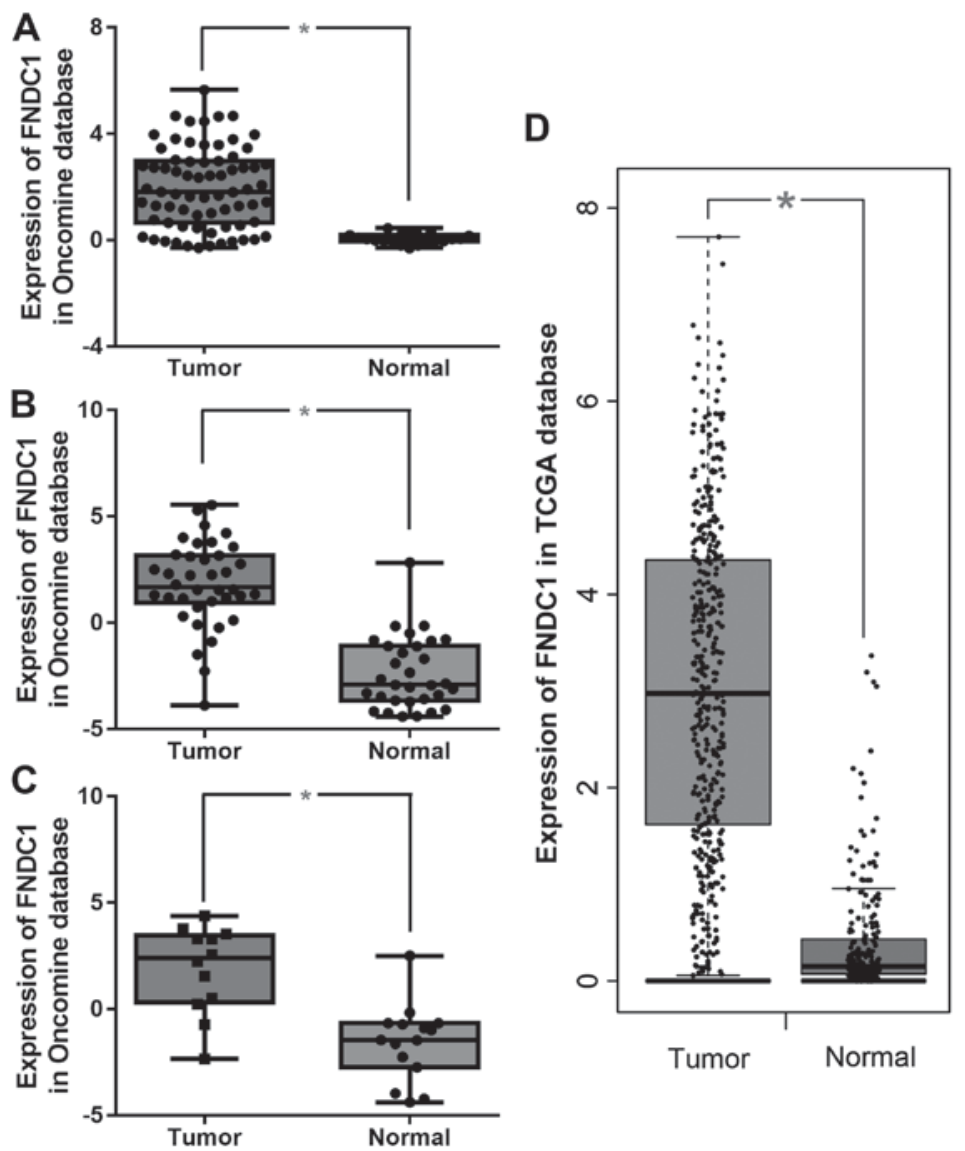

Figure 1. FNDC1 is upregulated in gastric cancer. FNDC1 expression in gastric cancer and normal tissues from the following datasets from Oncomine: (A) Cho, (B) Derrico and (C) Wang. (D) FNDC1 expression in gastric cancer and normal tissues from The Cancer Genome Atlas database analyzed by GEPIA. ${ }^{*} \mathrm{P}<0.01$. FNDC1, fibronectin type III domain containing 1.
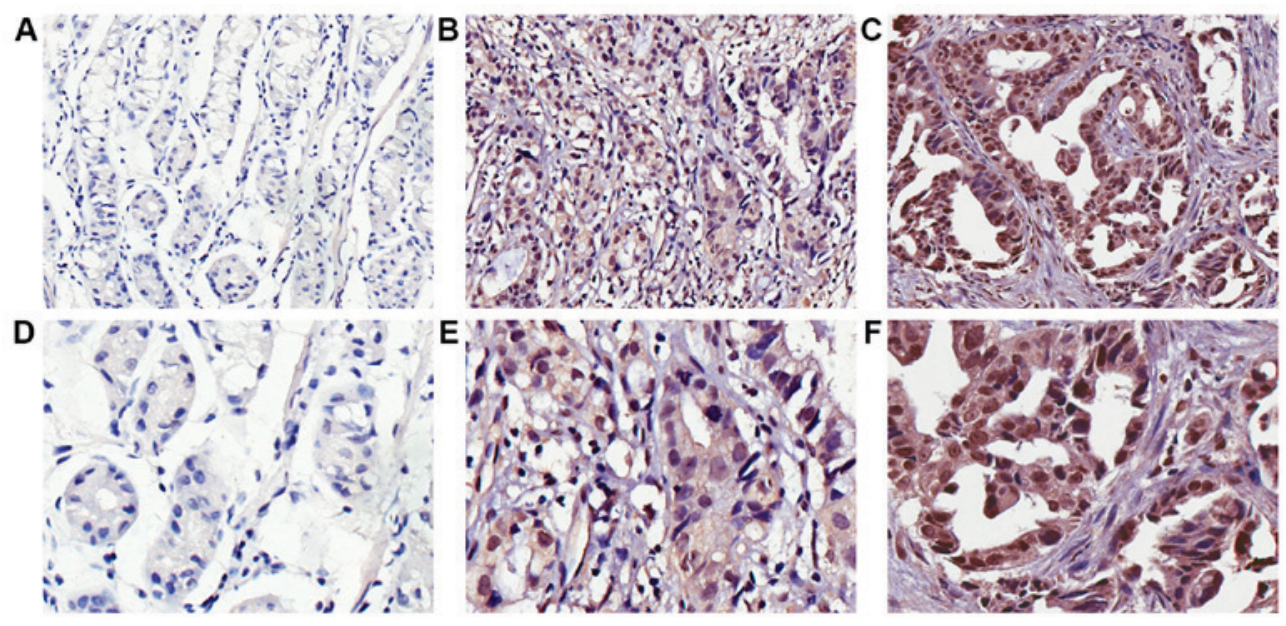

Figure 2. Immunohistochemical staining of FNDC1 expression in gastric cancer and adjacent normal tissue. (A and D) Adjacent normal tissues show negative FNDC1 protein expression; (B and E) Gastric cancer tissues exhibit different levels of low FNDC1 expression and (C and F) high FNDC1 expression level. Original magnification, x200 in A-C; x400 in D-F. FNDC1, fibronectin type III domain containing 1.

between FNDC1 expression and clinical stage $(\mathrm{P}<0.001)$, $\mathrm{T}$ classification $(\mathrm{P}<0.001), \mathrm{N}$ classification $(\mathrm{P}<0.001)$ and pathological differentiation $(\mathrm{P}=0.044)$. However, the expression of FNDC1 was not associated with age $(\mathrm{P}=0.467)$, sex $(\mathrm{P}=0.17)$, tumor size $(\mathrm{P}=0.668)$, or metastasis $(\mathrm{P}=0.084)$. These results indicated a significant association between FNDC1 expression and the prognosis of gastric cancer.
High FNDC1 expression in gastric cancer tissues correlates with poor patient survival. Cox regression analysis was used to determine whether FNDC1 expression could serve as a risk factor. As presented in Table II, using univariate Cox regression analyses, it was demonstrated that high FNDC1 expression level was associated with a significantly increased risk of death in patients with gastric cancer $(\mathrm{P}=0.001)$ compared with that in 
Table II. Univariate and multivariate analyses of various prognosis parameters in 98 patients with gastric cancer using Cox regression model.

\begin{tabular}{|c|c|c|c|c|c|}
\hline \multirow[b]{2}{*}{ Variable } & \multirow[b]{2}{*}{ Cases (n) } & \multicolumn{2}{|c|}{ Univariate analysis } & \multicolumn{2}{|c|}{ Multivariate analysis } \\
\hline & & P-value & HR $(95 \%$ CI $)$ & P-value & HR $(95 \%$ CI $)$ \\
\hline \multicolumn{6}{|l|}{ Age (years) } \\
\hline$<60$ & 53 & 0.413 & $1.246(0.736-2.110)$ & & \\
\hline$\geq 60$ & 45 & & & & \\
\hline \multicolumn{6}{|l|}{ Sex } \\
\hline Male & 74 & 0.118 & $1.593(0.889-2.854)$ & & \\
\hline Female & 24 & & & & \\
\hline \multicolumn{6}{|c|}{ Tumor size (diameter in $\mathrm{cm}$ ) } \\
\hline$<5$ & 51 & 0.028 & $1.811(1.065-3.078)$ & 0.056 & $1.684(0.986-2.877)$ \\
\hline$\geq 5$ & 47 & & & & \\
\hline \multicolumn{6}{|l|}{ Clinical stage } \\
\hline I & 7 & $<0.001$ & $2.343(1.524-3.603)$ & 0.031 & $2.907(1.105-7.653)$ \\
\hline II & 11 & & & & \\
\hline III & 62 & & & & \\
\hline IV & 18 & & & & \\
\hline \multicolumn{6}{|l|}{$\mathrm{T}$ classification } \\
\hline $\mathrm{T} 1+\mathrm{T} 2$ & 11 & 0.122 & $2.507(0.783-8.024)$ & & \\
\hline $\mathrm{T} 3+\mathrm{T} 4$ & 87 & & & & \\
\hline \multicolumn{6}{|l|}{$\mathrm{N}$ classification } \\
\hline No & 24 & 0.026 & $1.299(1.031-1.635)$ & 0.861 & $0.977(0.752-1.269)$ \\
\hline $\mathrm{N} 1$ & 16 & & & & \\
\hline $\mathrm{N} 2$ & 15 & & & & \\
\hline $\mathrm{N} 3$ & 43 & & & & \\
\hline \multicolumn{6}{|l|}{ Metastasis } \\
\hline No & 80 & 0.016 & $2.124(1.150-3.924)$ & 0.370 & $0.592(0.188-1.862)$ \\
\hline Yes & 18 & & & & \\
\hline \multicolumn{6}{|c|}{ Pathologic differentiation } \\
\hline Well & 5 & 0.041 & $1.732(1.022-2.936)$ & 0.262 & $1.355(0.797-2.306)$ \\
\hline Moderate & 32 & & & & \\
\hline Poor & 61 & & & & \\
\hline FNDC1 expression & & 0.001 & $3.543(1.667-7.533)$ & 0.032 & $2.326(1.073-5.038)$ \\
\hline Low & 27 & & & & \\
\hline High & 71 & & & & \\
\hline
\end{tabular}

HR, hazard ratio; CI, confidence interval; FNDC1, fibronectin type III domain containing 1.

patients with low FNDC1 expression level. Using multivariate Cox regression analysis, it was also determined that FNDC1 could be an important factor for predicting poor survival when FNDC1 expression $(\mathrm{P}=0.032)$ and clinical stage were included $(\mathrm{P}=0.031$; Table II). Patients with high FNDC1 expression levels had shorter overall survival (OS) times compared with patients with low FNDC1 expression levels (Fig. 3). To further understand the association between survival and FNDC1 expression in gastric cancer, the correlation between FNDC1 expression level and the survival of patients with gastric cancer was evaluated using the Kaplan-Meier Plotter (Fig. 4) and it was demonstrated that low FNDC1 expression level is a favorable prognostic factor for OS and post-progression survival (PPS; $\mathrm{P}<0.001, \mathrm{n}=631 ; \mathrm{P}<0.001$, $\mathrm{n}=384$, respectively) in patients with gastric cancer. Collectively, these results indicated that overexpression of FNDC1 in patients with primary gastric cancer is correlated with poor survival.

\section{Discussion}

Gastric cancer is a common malignant neoplasm that poses a serious threat to human life and health. Hence, it is considerably important to investigate the pathogenesis of gastric cancer and identify highly sensitive and specific molecular biomarkers for gastric cancer. Fibronectin is an important ECM protein that 
A

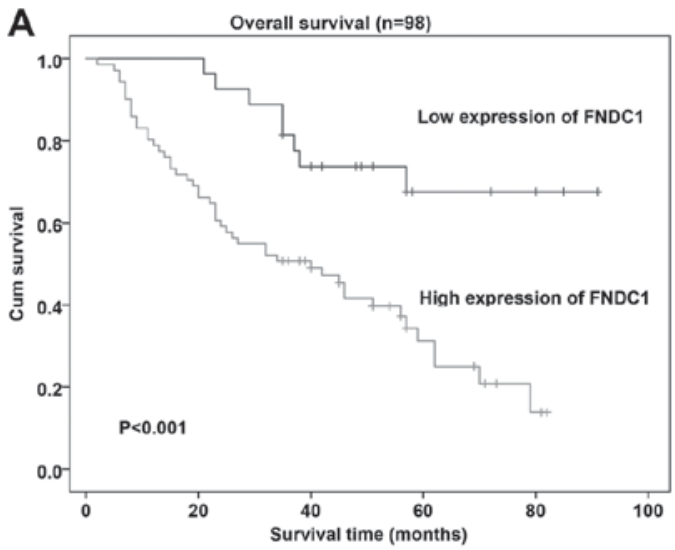

B

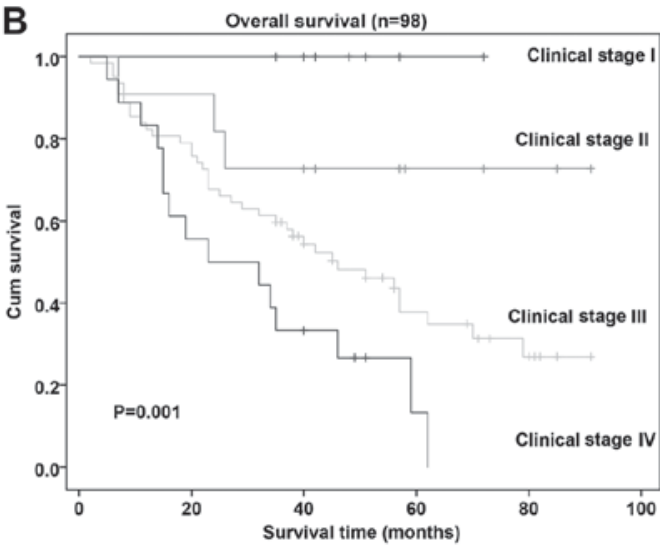

Figure 3. Survival curves of gastric cancer by the Kaplan-Meier method and the log-rank test. (A) Overall survival curves of high FNDC1 expression and low FNDC1 expression. (B) Overall survival curves by clinical stage I, clinical stage II, clinical stage III, and clinical stage IV. FNDC1, fibronectin type III domain containing 1 ; cum, cumulative.
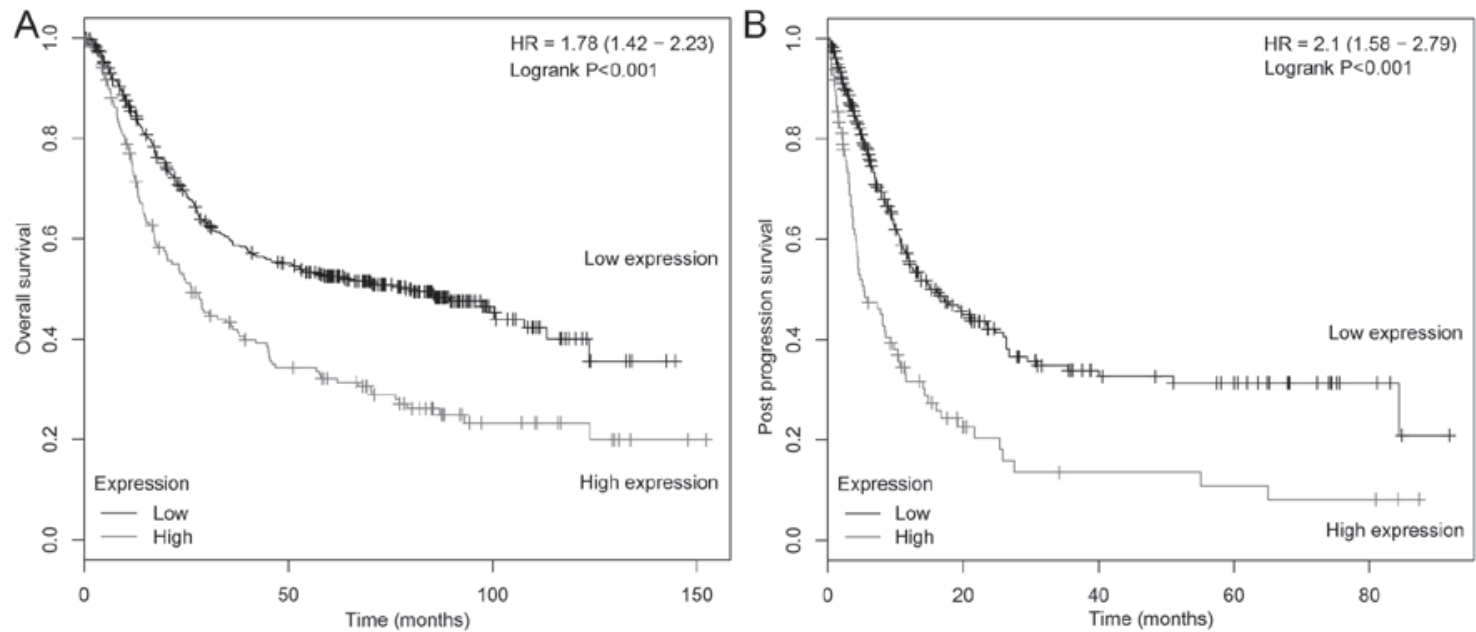

Figure 4. Prognostic value of FNDC1 expression in patients with gastric cancer. FNDC1 probe number is 226930_at. Results were analyzed using the Kaplan-Meier Plotter database. High expression of FNDC1 was associated with lower (A) OS and (B) PPS. FNDC1, fibronectin type III domain containing 1; HR, hazard ratio; OS, overall survival; PPS, post-progression survival.

has been reported to promote invasiveness of gastric cancer cells, and the serum fibronectin levels of patients with gastric cancer were significantly higher than those in the healthy controls $(32,33)$. FNDC1 contains a major component of the structural domain of fibronectin (10-13). The present study indicated that FNDC1 expression is higher in gastric cancer tissues than in adjacent normal tissues. FNDC1 may serve a role in tumor invasion in gastric cancer, as there were significant associations between FNDC1 expression and the depth of tumor invasion, lymph node metastasis, clinical stage and poor differentiation. Consistent with the present results, Oncomine and TCGA database analyses demonstrated that FNDC1 was overexpressed in gastric cancer tissues. Furthermore, it was observed that high FNDC1 protein expression was an independent prognostic factor for gastric cancer and was significantly correlated with poor survival. Bioinformatic databases were also used to confirm the association between FNDC1 expression and prognosis in gastric cancer patients. Results obtained using the Kaplan-Meier Plotter verified the finding that high FNDC1 expression was associated with poor OS and PPS in patients with gastric cancer.
Only limited data have been reported regarding the function of FNDC1. Chronic inflammation is a well-documented risk factor in cancer development (34-36). FNDC1 was initially identified as a desmoplastic response-related gene and seemed to have a role in inflammation (14). FNDC1 expression was correlated with skin tumor progression and could be induced by treatment with transforming growth factor- $\beta$, interleukin- 1 , and tumor necrosis factor- $\alpha$ in vitro (14). Notably, $12 \%$ of normal adjacent tissues in the present study exhibited positive FNDC1 staining. These tissues were demonstrated to exhibit atrophic gastritis and low-grade dysplasia. This suggested that the positive staining of these tissues may be associated with the malignant progression of gastric tissue. A previous study on acute otitis media in children identified that associated variants were significantly correlated with the expression levels and methylation status of FNDC1, and mouse homolog Fndc1 was upregulated under proinflammatory conditions, such as in lipopolysaccharide treatment (17). FNDC1 has been demonstrated to have an important role in the regulation of proliferation, apoptosis, and migration in prostate cancer (16). Previous studies have also reported that FNDC1 was expressed 
in kidney and heart tissue and served a role in hypoxia-induced apoptosis of cardiomyocytes by interacting with $\mathrm{G} \beta \gamma 38$ (18). FNDC1-G $\beta \gamma$ signal input also affects the function of CX43 and cell permeability, thus increasing the sensitivity of cells tohypoxic stress (19). Angiogenesis serves a critical role in malignant tumor growth and metastasis and is regulated by proangiogenic and antiangiogenic factors (37). VEGF is associated with physiological and pathological angiogenesis, which enhances the permeability of blood vessels, reduces endothelial cell apoptosis, activates stromal proteolysis, and promotes the proliferation and migration of endothelial cells $(38,39)$. Recently, Hayashi et al (21) demonstrated that knockdown of FNDC1 in endothelial cells inhibited VEGF-mediated cellular events, including cell growth, migration and proliferation. These results may be analogous to the results of the present study of gastric cancer. FNDC1 seems to have an important role in cellular proliferation and angiogenesis, which are necessary for tumor growth and metastasis.

To the best of our knowledge, this is the first study to investigate the association between FNDC1 expression levels and the clinicopathological features of patients with gastric cancer. Expression of FNDC1 was associated with unfavorable clinical characteristics and poor prognosis of patients with gastric cancer, suggesting that FNDC1 may have a positive regulatory role in gastric cancer by acting as a tumor-promotor gene. However, the present study is a retrospective observational study, and the results may not be representative of other gastric cancer populations. Further studies are required to determine the potential function of FNDC1 expression in tumor invasion and metastasis to verify the molecular basis of FNDC1 expression in gastric cancer. In addition, research on the changes in fibronectin levels following treatment of patients with gastric cancer should be performed in future studies.

In conclusion, the present results demonstrated that high FNDC1 expression level in human gastric cancer is significantly correlated with the progression as well as poor prognosis of the tumor. The present findings support the possibility that FNDC1 expression levels may be an important prognostic indicator and potential therapeutic target in gastric cancer.

\section{Acknowledgements}

Not applicable.

\section{Funding}

The present study was supported by the Natural Science Foundation of Guangdong Province (grant no. 2017A030313472).

\section{Availability of data and materials}

All data generated or analyzed during this study are included in this published article.

\section{Authors' contributions}

MZ and YiZ contributed equally to this study. MZ assisted in the design of the study, performed experiments, analyzed data and drafted the manuscript. YiZ contributed to the study design, interpreted data, and helped with the manuscript revision. FY and YP contributed to data analysis and helped draft and revise the manuscript. JW and JY provided technical support and assisted with the manuscript revision. WZ contributed to the study design and helped draft the manuscript. YaZ contributed to the study design, helped revise the manuscript and provided funding. All authors read and approved the final manuscript.

\section{Ethics approval and consent to participate}

The collection of tissue samples used for this study was approved by the Ethics Committee of Nanfang Hospital (Guangzhou, China) and all patients provided informed written consent.

\section{Patient consent for publication}

All participants gave informed written consent prior to taking part in this study. All samples were anonymized. Identifying information, including names, initials, date of birth or hospital numbers, images or statements were not included in the manuscript.

\section{Competing interests}

The authors declare that they have no competing interests.

\section{References}

1. Shah MA and Ajani JA: Gastric cancer-an enigmatic and heterogeneous disease. JAMA 303: 1753-1754, 2010.

2. Van Cutsem E, Sagaert X, Topal B, Haustermans K and Prenen H: Gastric cancer. Lancet 388: 2654-2664, 2016.

3. Chen W, Zheng R, Baade PD, Zhang S, Zeng H, Bray F, Jemal A, $\mathrm{Yu}$ XQ and He J: Cancer statistics in China, 2015. CA Cancer J Clin 66: 115-132, 2016.

4. Suzuki H, Iwasaki E and Hibi T: Helicobacter pylori and gastric cancer. Gastric Cancer 12: 79-87, 2009.

5. Kato S, Tsukamoto T, Mizoshita T, Tanaka H, Kumagai T, Ota H, Katsuyama T, Asaka M and Tatematsu M: High salt diets dose-dependently promote gastric chemical carcinogenesis in Helicobacter pylori-infected Mongolian gerbils associated with a shift in mucin production from glandular to surface mucous cells. Int J Cancer 119: 1558-1566, 2006.

6. Milne AN, Carneiro F, O'Morain C and Offerhaus GJ: Nature meets nurture: Molecular genetics of gastric cancer. Hum Genet 126: 615-628, 2009.

7. Gomceli I, Demiriz B and Tez M: Gastric carcinogenesis. World J Gastroenterol 18: 5164-5170, 2012.

8. Hartgrink HH, Jansen EP, van Grieken NC and van de Velde CJ: Gastric cancer. Lancet 374: 477-490, 2009.

9. Wagner AD and Moehler M: Development of targeted therapies in advanced gastric cancer: Promising exploratory steps in a new era. Curr Opin Oncol 21: 381-385, 2009.

10. Cao Y, Liu X, Lu W, Chen Y, Wu X, Li M, Wang XA, Zhang F, Jiang L, Zhang Y, et al: Fibronectin promotes cell proliferation and invasion through mTOR signaling pathway activation in gallbladder cancer. Cancer Lett 360: 141-150, 2015.

11. Fernandez-Garcia B, Eiró N, Marín L, González-Reyes S, González LO, Lamelas ML and Vizoso FJ: Expression and prognostic significance of fibronectin and matrix metalloproteases in breast cancer metastasis. Histopathology 64: 512-522, 2014.

12. von Au A, Vasel M, Kraft S, Sens C, Hackl N, Marx A, Stroebel P, Hennenlotter J, Todenhöfer T, Stenzl A, et al: Circulating fibronectin controls tumor growth. Neoplasia 15: 925-938, 2013.

13. Gao M, Craig D, Lequin O, Campbell ID, Vogel V and Schulten K: Structure and functional significance of mechanically unfolded fibronectin type III1 intermediates. Proc Natl Acad Sci USA 100: 14784-14789, 2003. 
14. Anderegg U, Breitschwerdt K, Köhler MJ, Sticherling M, Haustein UF, Simon JC and Saalbach A: MEL4B3, a novel mRNA is induced in skin tumors and regulated by TGF-beta and pro-inflammatory cytokines. Exp Dermatol 14: 709-718, 2005.

15. Bell A, Bell D, Weber RS and El-Naggar AK: CpG island methylation profiling in human salivary gland adenoid cystic carcinoma. Cancer 117: 2898-2909, 2011.

16. Das DK, Naidoo M, Ilboudo A, Park JY, Ali T, Krampis K, Robinson BD, Osborne JR and Ogunwobi OO: miR-1207-3p regulates the androgen receptor in prostate cancer via FNDC1/fibronectin. Exp Cell Res 348: 190-200, 2016.

17. van Ingen $\mathrm{G}, \mathrm{Li} \mathrm{J}$, Goedegebure A, Pandey $\mathrm{R}, \mathrm{Li}$ YR, March ME, Jaddoe VW, Bakay M, Mentch FD, Thomas K, et al: Genome-wide association study for acute otitis media in children identifies FNDC1 as disease contributing gene. Nat Commun 7 12792, 2016.

18. Sato M, Cismowski MJ, Toyota E, Smrcka AV, Lucchesi PA, Chilian WM and Lanier SM: Identification of a receptor-independent activator of G protein signaling (AGS8) in ischemic heart and its interaction with Gbetagamma. Proc Natl Acad Sci USA 103: 797-802, 2006.

19. Sato M, Jiao Q, Honda T, Kurotani R, Toyota E, Okumura S, Takeya T, Minamisawa S, Lanier SM and Ishikawa Y: Activator of G protein signaling 8 (AGS8) is required for hypoxia-induced apoptosis of cardiomyocytes: Role of G betagamma and connexin 43 (CX43). J Biol Chem 284: 31431-31440, 2009.

20. Liu CT, Garnaas MK, Tin A, Kottgen A, Franceschini N, Peralta CA, de Boer IH, Lu X, Atkinson E, Ding J, et al: Genetic association for renal traits among participants of African ancestry reveals new loci for renal function. PLoS Genet 7 : e1002264, 2011

21. Hayashi H, Al Mamun A, Sakima M and Sato M: Activator of G-protein signaling 8 is involved in VEGF-mediated signal processing during angiogenesis. J Cell Sci 129: 1210-1222, 2016.

22. Bosman FT, Carneiro F, Hruban RH and Theise ND: WHO classification of tumours of the digestive system. 4th ed. International Agency for Research on Cancer, Lyon, 2010.

23. Amin MB, Edge S, Greene FL, Schilsky RL, Gaspar LE, Washington MK, Sullivan DC, Brookland RK, Brierley JD, Balch CM, et al (eds.): AJCC cancer staging manual. 8th ed. Springer, New York, 2017.

24. Cho JY, Lim JY, Cheong JH, Park YY, Yoon SL, Kim SM, Kim SB, Kim H, Hong SW, Park YN, et al: Gene expression signature-based prognostic risk score in gastric cancer. Clin Cancer Res 17: 1850-1857, 2011.

25. D'Errico M, de Rinaldis E, Blasi MF, Viti V, Falchetti M, Calcagnile A, Sera F, Saieva C, Ottini L, Palli D, et al: Genome-wide expression profile of sporadic gastric cancers with microsatellite instability. Eur J Cancer 45: 461-469, 2009.

26. Wang Q, Wen YG, Li DP, Xia J, Zhou CZ, Yan DW, Tang HM and Peng ZH: Upregulated INHBA expression is associated with poor survival in gastric cancer. Med Oncol 29: 77-83, 2012.
27. Tang Z, Li C, Kang B, Gao G, Li C and Zhang Z: GEPIA: A web server for cancer and normal gene expression profiling and interactive analyses. Nucleic Acids Res 45 (W1): W98-W102, 2017.

28. Wan J, Liu H, Feng Q, Liu J and Ming L: HOXB9 promotes endometrial cancer progression by targeting E2F3. Cell Death Dis 9: 509, 2018.

29. Wang Y, Fu D, Chen Y, Su J, Wang Y, Li X, Zhai W, Niu Y, Yue D and Geng H: G3BP1 promotes tumor progression and metastasis through IL-6/G3BP1/STAT3 signaling axis in renal cell carcinomas. Cell Death Dis 9: 501, 2018.

30. Yang Z, Yang F, Zhang Y, Wang X, Shi J, Wei H, Sun F and Yu Y: Girdin protein: A potential metastasis predictor associated with prognosis in lung cancer. Exp Ther Med 15: 2837-2843, 2018.

31. Liu Y, Yao X, Zhang Q, Qian L, Feng J, Bian T, Zhang J and Tian Y: Expression of Kruppel-like factor 8 and Ki67 in lung adenocarcinoma and prognosis. Exp Ther Med 14: 1351-1356, 2017.

32. Li D, Ding J, Wang X, Wang C and Wu T: Fibronectin promotes tyrosine phosphorylation of paxillin and cell invasiveness in the gastric cancer cell line AGS. Tumori 95: 769-779, 2009.

33. Tas F, Bilgin E, Karabulut S, Tastekin D and Duranyildiz D: Levels of serum fibronectin as a biomarker in gastric cancer patients: Correlation with clinical diagnosis and outcome. Mol Clin Oncol 4: 655-659, 2016.

34. O'Byrne KJ and Dalgleish AG: Chronic immune activation and inflammation as the cause of malignancy. Br J Cancer 85: 473-483, 2001

35. Coussens LM and Werb Z: Inflammation and cancer. Nature 420: 860-867, 2002

36. Aggarwal BB, Shishodia S, Sandur SK, Pandey MK and Sethi G: Inflammation and cancer: How hot is the link? Biochem Pharmacol 72: 1605-1621, 2006.

37. Poon RT, Fan ST and Wong J: Clinical implications of circulating angiogenic factors in cancer patients. J Clin Oncol 19 1207-1225, 2001.

38. Tischer E, Gospodarowicz D, Mitchell R, Silva M, Schilling J, Lau K, Crisp T, Fiddes JC and Abraham JA: Vascular endothelial growth factor: A new member of the platelet-derived growth factor gene family. Biochem Biophys Res Commun 165 : 1198-1206, 1989.

39. Kowanetz M and Ferrara N: Vascular endothelial growth factor signaling pathways: Therapeutic perspective. Clin Cancer Res 12: 5018-5022, 2006. 